\title{
Integralidade: Desafio Pedagógico do PET-Saúde /UFC
}

\section{Comprehensiveness: a Pedagogical Challenge for the Educational Program for Health Work/UFC}

\author{
Paulo Sérgio Dourado Arrais \\ Andréa Silvia Walter de Aguiar \\ Miguel Ângelo Nobre e Souzal \\ Márcia Maria Tavares Machado \\ Maria Vaudelice Mota \\ Renata de Sousa Alves ${ }^{I}$ \\ Maria Fátima Maciel Araújo
}

PALAVRAS-CHAVE:

- Atenção Básica;

- Integralização;

- Multidisciplinaridade.

\section{KEYWORDS:}

- Primary Care

- Integrity

- Interdisciplinary.
Recebido em: 24/09/2010

Aprovado em: 19/11/2010

\begin{abstract}
RESUMO
A inserção dos sujeitos nos cenários de prática na Atenção Básica tem sido apontada como uma experiência instigante,poisreúne professores, estudantes, profissionais de saúde, gestores e comunidade, desafiando agregar valores, conhecimentos e experiência, a partir do caráter de interdisciplinaridade e integralidade das ações pedagógicas.Os avanços se relacionam na existência de um projeto, o PET-Saúde/UFC, com proposta metodológica de significativa operacionalidade para integralidade e interdisciplinaridade para os cursos de graduação em saúde.O presente trabalho relata as estratégias pedagógicas que fomentaram a compreensão do cuidado na Atenção Básica como um todorealizadas pelo projeto por meiodo desenvolvimento de um percurso metodológico para percepçãodo vivido e da totalidade, enquanto relato de experiência do movimento em construção de processos formativos.
\end{abstract}

\section{ABSTRACT}

Practical activities in primary care have been identified as a fulfilling experience, to the extent that they convene professors, students, health professionals, administrators, and the community, who are challenged to add values, knowledge, and experience based on the interdisciplinary and comprehensive nature of the teaching activities. Progress in this area has been identified in the Educational Program for Health Work at UFC, as an efficient methodological proposal for comprehensive, interdisciplinary learning in undergraduate health courses. The current study reports on the pedagogical strategies that fostered an understanding of primary care as a whole through the development of a methodological approach for grasping the total living experience, as a movement in the construction of formative processes. 


\section{INTRODUÇÃO}

A sintetizaçãodos esforços empreendidos na construção da integralidadepela equipe de coordenação do projetoPET-Saúde / UFC revela-se uma prática de formação da graduação em saúde. A universidade tem sido um lugar de incipiência na discussão da formação da graduação. Verifica-seque tratar dessa temática não é uma prática simples de ser construída, mas reputa-se como desafiante, por se apresentar como elemento substancialpara os debates educacionais proporcionados pelas ações do PET-Saúde,que reverberam em todos os espaços universitários. O que se tem tornado mais claro para todos os envolvidos nesse processo de reorientação da formação da graduação em saúde é que a formação ultrapassa o contexto da sala de aula, não se restringindo apenas ao tratamento de questões convencionais, como currículo, projeto pedagógico ou processos de avaliação. O objeto desses instrumentos de organização dos processos formativos só faz sentido quando pensado dentro de contextos mais amplos ou daquilo que a sociedade considera conquista para garantia dos direitos da sociedade.

Nessa interface reflexiva, é reconhecido, nos propósitos e práticas operacionais do projeto PET-Saúde/UFC, contornos desafiantese de muitas implicações, ao ultrapassar os limites da interface individual para o grupal-coletivo e na compreensão de que todos os espaços do sistema são espaços privilegiados de desenvolvimento de experiência pedagógica.As instituições parceiras (Universidade Federal do Ceará e Sistema Municipal de Saúde Escola da Secretaria de Saúde do Município de Fortaleza)fazem um exercício de assumircompromissospara enfrentar os problemas, resolver conflitos e cultivar a cultura de abertura aos outros, necessária para desenvolver a coragem, a competência e habilidades para alimentar propostas fortalecidas em abordagens mais participativas consolidadas nas parcerias, na reciprocidade e no rigor científico. Esse caminho pode oferecer um conjunto de ferramentas e possibilidades para pensar concepções de saúde, formação e integralidade a partir dos sujeitos envolvidos e suas concepções de mundo.

Nesse exercício,tem sido possível a experimentação de ações assistivas/cuidativas fomentadas em tecnologias de abrangências ampliadasque influenciama alteração de objetivos de formação, para odeslocamento também dodesenvolvimento do pensamento crítico.Essemovimentofez e faz emergir uma ação desafiadora nas instituições parceiras, que é a de compreender as ações do PET-Saúdecomo integrantes de um movimento técnico-político-institucional que vem envolvendo vários sujeitos em todos os momentos e espaços da aprendizagem. Nesse movimento, observa-se uma "rede integrati- va docente", que,sutilmente, organiza-se, dando visibilidade a ações pedagógicas mais colaborativasnos cenários de prática e no campo da pesquisa, criando fóruns como espaços de debate e análise mais profunda dos processos formativos. Há de se reconhecer, no entanto, que há anecessidade de maiores investimentos para seu refinamento enquanto estratégia auxiliadorados processos de formação da graduação dos cursos de saúde.

A estratégia de rodas, que expressa o modo de operação do PET-Saúde/UFC, é outra ferramenta usada como estratégia de organização e gestão do projeto que tem sido relevante ao proporcionar o encontro entre os diferentes cursos e as diferentes formas de operacionalizar os projetos pedagógicos. Promove, assim, a diversidade de saberes constituindo, principalmente, os processos criativos que vão sendo desvelados na elaboração dos pequenos projetos em desenvolvimento nas comunidades/áreas na Atenção Básica. Sem exprimir juízos para procurar avaliar o mérito acadêmico de cada projeto,os tutoresforam procurando conhecer a diversidade de ideias, contextualizando seus reflexos na atitude educativa que produz a formação libertadora na prática dos estudantes, pelo desejo do diálogo e relações sobre o que existe de fato entre a formação e a vida social. Esse percurso fomenta elementos que orientam as bases organizativas para a formação de profissionais de saúde na centralidade dos projetos pedagógicos de cada curso, dos sujeitos protagonizando seus próprios caminhos de aprendizagem, desenvolvendo competências técnicas e educação para o processo de trabalho em saúde, base para pensar a excelência técnica e preparação para o desempenho cívico.

Nessas formas de organização das experiências das rodasainda se enfrenta contradições, tais como a reprodução de velhos vícios, como a priorização de acordos sem a devida participação das partes, presença de pessoas/grupos de pouco diálogo. Apesar de serem articulados,por vezes esses antigos hábitos se flexibilizam gerando necessidades de se construir novas críticas e relações diante do desafio de uma nova formação que se pauta, essencialmente, no processo de organização político-educativo desenvolvido na parceria ensino-serviço.

Sob essa perspectiva, a experiência adquire concretude deflagrando inúmeras reflexões que se inserem em um amplo espectro de possibilidades teórico-práticas interessantes ao processo formador consolidado na presença e no movimento dinâmico provocado pelas ações do projeto. Não obstante, por seu valor estratégico, entende-se que a inteligência (aprender a aprender), os sentimentos (aprender a ser e a conviver), o ambiente ea natureza (aprender a fazer) atuam de forma interligada, e toda separação entre partes pode parecer uma abordagem redutora para o entendimento do processo formador. 
Com isso, objetiva-se relatar as estratégias pedagógicas que fomentam a integralidade do cuidado na Atenção Básica.

\section{METODOLOGIA}

O intuito é desenvolver um percurso metodológico para compreensão do vivido e indicar alguns elementos da integralidade fomentados por meio da ação enquanto narrativa, fruto da construção de processos formativos cujas informações foram reunidas por meio de relatórios e avaliações realizadas no decorrer do ano de 2009. A narrativa é uma alternativa metodológica que possibilita expressar um tempo e uma circunstância. Essa mesma metodologia considera inicialmente que, ao se utilizar o modo narrativo para relatar acontecimentos, eventos, situações, pode-seobter diversas interpretações,contudo, os elementos que constituem os problemas pensados sempre prevalecem no cerne do relato. Tal escolha considera a flexibilidade, possibilitando reunir a teoria com a vida cultural e social. Nessa construção, a técnica de produção colaborativa agrega outros componentes que possibilitam ao sujeito que narra estar envolvido na ação, compreendero movimento e interpretara realidade.

Sua relevância se manifesta na medida em que é indispensável que esses sujeitos tenham vivenciado a experiência e a queiram relatar. Essa possibilidade faz surgir a integração entre teoria e prática como um processo formativo crítico. A flexibilidade que métodos como esse oferece permite a sua razão instrumental em experiências desse porte, queconsidera a multiplicidade de ações a que estão submetidos os sujeitos em suas atividades docentes,sem que haja a necessidade de controles rígidos e formais para fomentar a organização e sistematização de práticas vivenciadas na Atenção Básica.

Segundo Holliday $(2004)^{1}$, a narrativa é um método que sistematiza experiências e se organiza em uma trajetória sequencial, contemplando começo, meio e fim; este entendido como sempre em movimento. A narrativa coloca o problema como centro e gira em torno de histórias, fatos, eventos, vivências que valem a pena e precisam ser contados. Em sua estrutura tem servido para provocardiscussões sobre a realidade e argumentos sobre a natureza das questões narradas; essa reflexão/ação articula a natureza da realidade e se transforma em argumentos epistemológicos, fornecendo uma base de construção de sentidos e significados sobre o vivido.

Para ilustrar o processo de sistematização de práticas e orientação da organização da narrativa, o quadro 1 apresenta palavras geradores sugestivas para descrição e exploração do processo metodológico articulado por meio das ações do projeto.

\section{QUADRO 1}

Representação da descrição das etapas de organização do relato de experiênciaPET-Saúde/UFC, 2009

\section{1 - COMPARTILHAMENTO}

Relato das experiências pedagógicas vividas pelos sujeitos: docentes, discentes, profissionais, trabalhadores de saúde, gestores, conselheiros.

\section{2 - EXTERNALIZAÇÃO}

Conversão da experiência pedagógica em conhecimento explícito, construindo visibilidade do movimento formativo.

3 - INTERNALIZAÇÃO

Incorporação do conhecimento em material pedagógico, textos, trabalhos científicos, relatórios, conteúdo/inclusão em cursos.

4 - SISTEMATIZAÇÃO

Sistematização de resultados, refinamento de propósitos

formativos, fortalecimento de parcerias, organização do núcleo de pesquisa.

Esse processo articulado pelos sujeitos participantes reunidos em torno de dezárvores tutoriais do PET-Saúde/UFC, no período de abril de 2009 a abril de 2010, envolvendo os cursosde graduação em Farmácia, Odontologia, Enfermagem, Medicina e Psicologia,,compreendendoum coordenador, dez tutores, 300 estudantes, 17 gestores de Centros de Saúde da Família, 60 preceptores e representantes do Sistema de Saúde Escola do Município de Fortaleza e do Conselho Municipal de Saúde.

\section{RESULTADO E DISCUSSÃO}

A respeito dessa produção,alguns registros foram reunidos, os quaisrevelam a significativa importância das ações do PET-Saúde na formação da graduação em saúde. Esses registros foram organizados em eixos temáticos, para que os discursos possam demonstraro significado das ações em seus vários aspectos.

Estreitamento e compreensão do Sistema Único de Saúde

$$
\begin{aligned}
& \text { "A experiência tem nos permitido vivenciar e compreender } \\
& \text { como ocorre a gestão do SUS." } \\
& \text { "O SUS parece mais próximo da gente e, por isso, vemos a } \\
& \text { distância entre a teoria e a prática." } \\
& \text { "O SUS é um grande projeto, mais ainda falta muito para } \\
& \text { sua consolidação como política pública de saúde." }
\end{aligned}
$$

\section{Envolvimento discente}
"Foi importante observar a importância da sala de situação para o profissional de saúde e para o gestor, e embasar o cida- dão no exercício do controle social."
"Com a oportunidade de realizar esta integração serviço- -academia... o contato com o Sistema de Saúde se consolida 
cada vez mais, promovendo o sentimento de cidadania e participação social."

"Necessidade de uma educação continuada para resolver as incertezas sobre tabus acerca das doenças."

"Contribuiu para o desenvolvimento de propostas educativas mais próximas da realidade."

"A territorialização permite constatar os problemas de saúde prevalentes e facilita a atuação do profissional que trabalha na Unidade Básica."

Os alunos buscam conhecer os serviços e se inserir na rotina de trabalho de uma Equipe de Saúde da Família."

"Estamos na fase de construção da sala de situação, elaborando as possíveis ações a serem desenvolvidas na comunidade."

"Entendemos que a sala de situação é um instrumento importante para a leitura da comunidade e compreensão de suas demandas, pois sistematiza informações e facilita a discussão entre os profissionais do serviço."

"A experiência ajuda a entender na prática como se organiza a saúde da família."

Melhoria do ensino de graduação, aproximação coma realidade social

"A ideia de um programa com apoio federal que estreita vínculos entre graduação e Atenção Básica já é por si só um enorme desafio...as atividades iniciais do Pet-Sáúde/UFC mostraram que o projeto tem um grande potencial para estimular a formação de profissionais realmente comprometidos com a Atenção Básica."

"Ressaltamos a vivência e o reconhecimento do território vivo útil para o conhecimento do aluno de graduação."

"Pode-se dialogar com a própria população sobre os problemas e as potencialidades da região."

\section{Importância da multidisciplinaridade na formação aca-} dêmica

\footnotetext{
"A prática oferece a troca de conhecimentos, mostrando a importância da prática multidisciplinar para a formação acadêmica."

"Fortalece a interdisciplinaridade, a integração de novos saberes, além da ampliação da reflexão crítica da inserção desses futuros profissionais no modelo de Atenção Básica."

"A proposta do Pet-Saúde/UFC permitiu uma maior inserção dos estudantes na comunidade e maior aprimoramento do trabalho em equipe."
}

Nessa vivência prática, os protagonistas do processo encontraram maneiras e estratégias de aprendizagem geradoras de muitas perguntas, dúvidas, questionamentos, assim como de algumas descobertas e respostas. Nela tem prevalecido o diálogo, a convivência com as diferenças e os diversos contextos entre saberes e fazeres da vida acadêmica cotidiana e a realidade social dos serviços e comunidades. Não foi destituída de relações polêmicas e conflituosas, fruto do incipiente exercício dessa construção no nível das instituições de serviços e instituições formadoras com propósitos tão desafiantes. Na experiência, uma nova densidade de prática educativa significativa se revela e vem sendo construída no "movimento teoria e prática".

A oportunidade de desenvolver habilidades instrumentais de cuidado pelos estudantes deu uma conotação de aprendizagem ativa e significativa. Foram realizadas ações como: territorialização (cadastro, levantamento de recursos disponíveis e rede social de apoio); acolhimento por gestão de risco; implementação de ações de prevenção e controle de DST /HIV-Aids e hepatites virais; prevenção e controle de hipertensão, diabetes, tuberculose e hanseníase; fortalecimento dos grupos (autoajuda, operativos, terapêuticos, grupos de encontro, grupo de salas de espera, orientação e vivência) junto aos serviços e à comunidade, tais como idosos, gestantes e adolescentes; discussão de estratégias na promoção do uso racional de medicamentos; desenvolvimento de experiências de cuidado com uso de práticas integrativas e complementares (massoterapia, cromoterapia, fitoterapia, entre outros); vigilância à saúde ambiental, epidemiologia e saúde do trabalhador; acompanhamento de ações preventivas relacionadas aos principais cânceres (câncer de mama, colo de útero e de boca); ações de promoção e prevenção em saúde bucal,como: controle de cárie dental, escovação, fluoterapia; participação em campanhas;orientação pararedução da mortalidade infantil, neonatal e materna;ações de prevenção das doenças imunopreveníveis; desenvolvimento de ações de saúde escolar; incentivo ao aleitamento materno exclusivo e prática de alimentação saudável; acompanhamento do crescimento e desenvolvimento na infância; prevenção de acidentes domésticos, como quedas, corpo estranho e queimaduras;controle da dengue e fomento de experiências e intervenção no domicílio.

Tudo isso tem contribuído de forma efetiva para a consolidação do conceito de integralidade do processo e vivência prática das experiências.Integralidade, no contexto do Sistema Único de Saúde ${ }^{2}$, pode ser vista como uma imagem objetiva, uma noção amálgama, com vários sentidos. A integralidade pode ser entendida como crítica às práticas dos profissionais de saúde, como uma dimensão que busca compreender o conjunto das necessidades de ações e serviços de 
saúde que incorpora a promoção na atenção à saúde.Pode ser percebida aindacomo modo de organizar o processo de trabalho em saúdee otimizar o seu impacto epidemiológico como oferta de um programa de atenção à saúde.Esse movimento tem tornando visível a capilaridade que processos como esses tendem a demandar, viabilizando operacionalmente relações institucionais e interinstitucionais entre ensino, serviço e comunidade.

O caráter da multiprofissionalidade e interdisciplinaridade se concretizam na proposta do projeto, na facilitação do acesso de um contingente de trabalhadores de saúde e estudantes das várias áreas e recursos de formação e educação permanente em condições reais de trabalho. As ações assentadas na eminência do diálogo enfatizam a dispersão de poder ao articular os sujeitos na problematização coletiva dos processos de formação, entendendo, porém, que a ampliação dos cenários de prática e inserção do aluno na Atenção Básica não garante por si só uma formação plena.

Compreende-se que a problematização da realidade tem limites que se concretizam na necessidade da visibilidade do objeto de trabalho das profissões de saúde na Atenção Básica, das novas competências e habilidades delas e na minimização da ansiedade clínica dos sujeitos, que repousa particularmente na dificuldade em garantir fluxos de cuidados progressivos e integrais à saúde da família por meio dos devidos encaminhamentos (referências/contrarreferências) e soluções dos problemas.

A discussão sobre a integralidade das ações de saúde também sugere iniciativas de avaliação que assumem caráter plural nos processos formativos historicamente centrados em modelos pedagógicos conteudistas. ${ }^{3,4}$ Com o intuito de defla- grar a avaliação das experiências do PET-Saúde/UFC nos processos formativos, apresenta-se alguns elementos orientadores como possíveis indicadores a serem trabalhados na reorientação dos processos formadores: poder de presença;poder de gestão;poder de conhecimento; epoder de fazer (QUADRO 2). O ponto de partida contemplado nesta intenção é de conceber modos mais instrumentais de avaliação com base no desenvolvimento de competências e habilidades como um exercício de construção de empoderamentoindividual e coletivo-grupal.

\section{CONCLUSÃO}

Foi constatado o envolvimento das instituições parceiras e protagonistas do processo dentro desse grande movimento de ressignificação da formação da graduação em saúde no município de Fortaleza.Nessa estratégia pedagógica, já se pode vislumbrar uma primeira síntese cuja natureza pode ser enfatizada na dimensão experiencial da estratégia desenvolvida de forma circular, que lembra os círculos de cultura propostos inicialmente por Paulo Freire. Essa proposta, em sua essência, já enfatiza uma relação imediata com práticas de ações cooperativas de gestão e como tema gerador formativo e fundamental nas profissões de saúde.

\section{REFERÊNCIAS}

1. Holliday OJ. Para sistematizar experiências. João Pessoa: Ed Universitária;1996.

2. Ministério da Saúde. Secretaria de Assistência à Saúde. Desenhos da Organização da Atenção no SUS. A Integralidade da Atenção à Saúde: Encontro dos estudantes universitários da área de saúde e o SUS. Brasília: MS;2003.

Quadro 2

Representação esquemática dos processos de avaliação do PET-Saúde/UFC.

\section{Poder de presença \\ Avalia a participação sistemática do aluno no território de saúde. \\ Mede a cobertura territorial e população de famílias cobertas, como \\ qualificação da presença. \\ Avalia a articulação com a comunidade e a rede social de apoio e a articulação com valores cívicos. \\ Avalia a capacidade para fazer escolhas e tomar decisões. \\ Instrumentos: fichas, prontuários, diário de campo, portfólio.Registros profissionais.}

\section{Poder de conhecimento}

Avalia a capacidade para construir diálogos entre teoria e prática, transferir e generalizar conhecimentos.

Avalia a capacidade de planejar processos de estudos, pesquisas e socialização de saberes.

Avalia a capacidade de realizar leitura de mundo e escrever clara e efetivamente sobre variados assuntos.

Instrumentos: capacidade de produção e publicação de trabalhos

científicos; participação em eventos acadêmicose grupos de pesquisa.

\section{Poder de gestão}

Avalia a participação nas rodas de gestão e atuação em grupo. Avalia capacidade para reconhecer problemas, equacionar soluções, introduzir modificações, gerenciando tempo,espaço informação. Avalia capacidade de elaborar projetos de intervenção e tecnologia de cuidado.

Instrumentos: diário de campo, portfólio, relatório.

\section{Poder de fazer}

Avalia a capacidade de realizar cuidados ampliados de saúde. Avalia a capacidade para executar procedimentos de cuidado com base naprática de excelência clínica

Avalia capacidade para elaboração de plano de cuidado à saúde da família. Avalia competências e habilidades de cuidados.

Instrumentos: prontuário, diário de campo, estudo de caso,relatório. Diário de campo. 
3. Pinheiro R, Matos RA. Os sentidos da integralidade na atenção e no cuidado à saúde. Rio de Janeiro: Abrasco; 2001.

4. Campos CEA. O desafio da integralidade segundo as perspectivas da vigilância da saúde e da saúde da família. Ciências Saúde Colet 2004;8(2): 569-84.

\section{CONTRIBUIÇÃO DOS AUTORES}

Os autores contribuíram na produção do trabalho com igual compromisso de tornar acessível aos leitores os resultados das experiências desenvolvidas no Pet-Saúde/UFC.

\section{CONFLITO DE INTERESSES}

Declarou não haver.

\section{ENDEREÇO PARA CORRESPONDÊNCIA}

Paulo Sérgio Dourado Arrais

Rua Alexandre Baraúna, 949

Rodolfo Teófilo - Fortaleza

CEP. 60430-160 CE

E-mail: parrais@ufc.br 